\title{
Cancer Immunothearapy More than Vaccines "Psychoneuro-Immunooncology: Cancer, the Host, and the Surgeon”
}

\section{Robert Lange Elliott}

Elliott-Elliott-Head Breast Cancer Research and Treatment Center, Baton Rouge, USA.

Email: relliott@eehbreastca.com

Received June $7^{\text {th }}, 2011$; revised July $4^{\text {th }}, 2011$; accepted July $11^{\text {th }}, 2011$.

\begin{abstract}
Cancer immunology is extremely complex with numerous interactions between the tumor and the host. It is time for those that treat cancer, especially surgeons, to learn more about these complex interactions. We need to know more about host immunity and immunosuppressive mechanisms which are not directly related to the disease, but caused by stress and therapy of the disease. The diagnosis of cancer initiates stress that can be very detrimental to the host immune system. Most cancer physicians (surgical, medical, and radiation oncologist) do not appreciate the impact on host cell mediated immunity (CMI) caused by cancer therapy, and definitely do not know how devastating, psychic stress is on host immunity. This communication is an attempt to bring awareness to this problem.
\end{abstract}

Keywords: Host Immunity, Cancer Immunosuppression, Cell Mediated Immunity, Psychic Stress

\section{Introduction}

Recently there has been a renewed interest in cancer immunotherapy especially in the area of cancer vaccine development. However, cancer immunology is extremely complex with numerous interactions between the tumor and the host. Therefore, it is time for those of us that treat cancer, especially surgeons, to learn more about these complex interactions. We definitely need to know more about host immunity and cancer immunosuppressive mechanisms, many which are not directly related to the disease, but caused by stress and treatment of the disease. This communication is an attempt to bring awareness to this problem.

\section{Discussion}

The diagnosis of cancer initiates a cascade of tremendous stressful events in the host of that disease. This stress can be very detrimental to the host immune system, which is probably already immunocompromised. Physicians treating cancer know it elicits fear, anger, anxiety, and depression, all very stressful, emotional events, which may impact the final outcome of the disease for the host. Most cancer physicians (surgical, medical, and radiation oncologist) do not appreciate the impact on host cell medi- ated immunity (CMI) caused by the therapies of cancer; and definitely do not know how devastating psychic stress is on host immunity.

In fact, host immunity in the cancer patient is totally ignored by most oncologists, especially in the private practice community setting [1]. Physician interest is mainly on the disease, type of tumor, tumor biology, clinical staging, and treatment options. During this stressful period, a devastating and possibly irreversible effect on the host immune system may occur. This immunosuppressive period may possibly determine the final treatment outcome and over-all survival for the host. Therefore, it is imperative that measures be implemented to reverse these immunosuppressive events immediately at the time of diagnosis.

The first and probably most important of theses measures is recognition of this very complex problem. The second is physician education about the complexity of host immunosuppression, and what steps can be taken to minimize or reverse these immunosuppressive events. These steps involve several phases requiring different approaches of immune support, but the support should start immediately at the time of diagnosis. The different phases to be addressed are: 1) at diagnosis, 2) during staging work-up, 3) consultation about treatment options, 
4) primary surgical treatment, 5) adjuvant therapy (hormonal, radiation, or chemotherapy) Table $\mathbf{1}$. The mechanisms of host immunosuppression may be different in each of the above phases, and each will require different approaches to support the host immune system. However, support should be implemented immediately and continue through each phase adjusting support based on the difference in the pathophysiology of each phase of host immune suppression. Supporting host immunity at the beginning of the first phase will condition the host immune system and allow for easier treatment support of host immunity through each phase of the cancer treatment protocol Table 2. Many of the statements in this communication are our opinion, and we now present evidence to support this opinion.

The relationship between the brain and the immune system is not appreciated by most oncologists; however, the brain and the immune system are the two major adaptive body systems. In 2000 Elenkov, Wilder, Chrousos, and Vizi [2] published a tremendous paper explaining in detail the relationship of these two major adaptive systems. They state that during an immune response the brain and immune system talk to each other and this is necessary for homeostasis. They show that the sympathetic nerve is an integrative interface between the two supersystems (the brain and the immune system).

Table 1. Stages of host cancer immunosuppression.

\begin{tabular}{ccc}
\hline A. & Escape from Immunosurveillance \\
\hline B. & Stress at Diagnosis \\
C. & Stress and Anxiety during Staging Work-up \\
D. & Stress Involved with Treatment Decisions \\
E. & Preoperative, Operative, and Post Operative Period \\
F. & 1. & Period of Adjuvant Treatment \\
& 2. & Chemotherapy \\
& 3. & Radiation \\
& (All stages affect host cancer immunosuppression by different mechanisms)
\end{tabular}

Table 2. Treatment strategies for host cancer immunosuppression.

\begin{tabular}{|c|c|c|}
\hline A. & & Diagnosis, Staging, and Treatment Decisions \\
\hline \multirow{7}{*}{ B. } & 1. & Treat anxiety—comfort patient, compassion, and treat with anti-anxiety drugs \\
\hline & 2. & Reverse catecholamine suppression with prostaglandin synthesis inhibitors and $\beta$-blockers \\
\hline & & Entire Perioperative Period \\
\hline & 1. & Evaluate pre-operative NK and T-cell numbers and activity \\
\hline & 2. & Continue A. 2. above \\
\hline & 3. & Timing of surgery especially in women during pre-menstrual and menstrual cycle \\
\hline & 4. & $\begin{array}{l}\text { Use Poly I-C to protect NK cells intraoperatively and post-operatively; also prophylactic use } \\
\text { of IL-12 and (CPG-CODN) }\end{array}$ \\
\hline \multirow{3}{*}{$\mathrm{C}$. } & 5 . & Address angiogenic growth factors associated with wound healing \\
\hline & & Post-Surgery Adjuvant Treatment? \\
\hline & 1. & Monitor NK cell and lymphocyte count during chemotherapy and radiation \\
\hline \multirow{8}{*}{ D. } & 2. & Consider host specific immunotherapy (autologous vaccine) \\
\hline & 3. & $\begin{array}{l}\text { Consider low dose intradermal IL-2 therapy based on lymphocyte count (May have to watch } \\
\text { FoxP3 T-Reg) }\end{array}$ \\
\hline & 4. & Monitor Th1 and Th2 cytokines especially IL-6 and IL6R, IL-17, IL-23, TGF-B, IL-10 \\
\hline & & After Adjuvant Therapy \\
\hline & 1. & Continue to monitor host immunity \\
\hline & 2 . & Support host immunity by reducing stress \\
\hline & 3. & Support host immunity with appropriate supplements and nutrition \\
\hline & 4. & Specific immunotherapy (various cancer vaccines) \\
\hline
\end{tabular}


The two major pathway systems involved in the crosstalk are the hypothalamic-pituitary-adrenal axis (HPA) and the sympathetic nervous system (SNS). Their overview focuses on the role of the (SNS) in neuroimmune interactions, which has not received the attention as has the role of the (HPA) axis.

Evidence over the last 20 years shows that norepinephrine (NE) fulfills the role for neurotransmitter/modulator in lymphoid organs. Primary and secondary lymphoid organs have extensive sympathetic noradrenergic innervations. When stimulated (NE) is released from sympathetic endings in these organs, and target immune cells expressing adrenoreceptors to respond. The locally released (NE), or circulating catecholamines affect lymphocyte circulation, proliferation and traffic. This modulates cytokine production and functional activity of different lymphoid cells.

They point out that there is strong evidence that (NE) and epinephrine through stimulation of the B2-adrenoreceptor-cAMP protein kinase A pathway inhibits production of Type I proinflammatory cytokines and stimulates the production of the Type 2 anti-inflammatory cytokines and thus, causes a selective suppression of the Th1 response and cellular mediated immunity (CMI) and shift toward dominance of a Th2 response and humeral immunity. A shift of a Th1 to a Th2 response in the tumor microenvironment promotes cancer progression. This phenomenon should be considered and addressed early in cancer treatment. Elenkov, et al. [2] state that in certain local conditions, catecholamines may boost regional immune responses through production of IL-1, tumor necrosis factor- $\mathrm{a}$ and production of IL-8. The (SNS) seems to be pro Th1 locally and pro Th2 systemically thus protecting the organism from detrimental effects of proinflammatory cytokines and products of activated macrophages. By utilizing this knowledge, one can pharmacologically manipulate the sympathetic immune interaction and focus on new therapeutic strategies for cancer treatment.

The above facts are undeniable, but no one has contributed more to the field of psychoneuroimmunology than Ben-Eliyahu and his group from Israel. In 2003, Ben-Eliyahu [3] reported on the promotion of tumor metastasis by surgery and stress. He laid the foundation for the immunological basis and implications for psychoneuroimmunology (PNI), which I have decided to expand to psychoneuro-immunooncology. His minireview emphasizes the (PNI) perspective and hypothesis that stress and surgical excision of the primary tumor can promote tumor metastasis. He establishes the theoretical basis for control of metastasis by (CMI) and the interactive role of non-immunological risk factors. He describes aspects of surgery that suppress (CMI) and neu- roendocrine mechanisms causing suppression by stress and surgery. Because of empirical evidence from animal and human studies, for promotion of metastasis by stress and surgery with special emphasis on the role of (CMI) it was concluded that: "1) Immunological mechanisms most likely play a role in limiting metastasis in patients with solid tumors. 2) Immunosuppression can be deleterious, especially when surgery is conducted early, before the tumor develops insurmountable mechanisms to escape immune destruction, 3) the most sensitive period for the establishment of metastases is the immediate aftermath of surgery. Interventions aiming at reducing stress and immunosuppression should thus strive to start beforehand. 4) Psychological and physiological insults activate similar neuroendocrine mechanisms of immunosuppression. Therefore, a multi-modal therapeutic approach should be used to prevent tumor metastasis during the peri-operative period. 5) Studies employing interventions arrived at reducing the surgical stress response should preferably assess immunological indices with established clinical relevance, and follow-up long time recurrence provided sample size assure statistical power. 6) The progress toward earlier detection of cancer, and our growing understanding of immunosuppression, continuously improves the chances for successful (PNI) interventions." The above conclusions are profound, but we totally agree with their concepts.

(CMI) probably begins as soon as a tumor presents and continues through the entire process of immunoediting [4]. After tumor escape and local tumor growth (CMI) may still contribute to systemic control of cancer by controlling macrometastatic disease and eradiating residual disease after surgical removal of the primary tumor. This is supported by the evidence from clinical studies reporting that immune competence at the time of cancer treatment is an independent prognostic indicator of disease free survival [5-7]. Non-immunological factors can promote metastasis after surgery. Malignant cells are loose in the stroma and close to blood vessels, surgery can release cells into the circulation $[8,9]$. The removal of the primary tumor promotes angiogenesis by eliminating tumor produced anti-angiogenic factors [10,11]. Wound repair after surgery releases growth factors that promote cancer progression and metastasis [12,13]. The tumor tissue should also be evaluated for the expression of Human Leukocyte Antigen G (HLA-G). We have recently reported on the role of HLA-G in breast cancer immunosuppression [14].

Major surgery suppresses (CMI) in animals and humans and the degree of immunosuppression correlates with the severity of tissue damage [15]. There are numerous cytokine responses to major surgery both locally and systemically. These are proinflammatory cytokines 
and many Type 2 immunosuppressive cytokines that interfere with (CMI) [16]. Melamed, Rosenne, Shakhar, et al. [17] have shown the suppressive effect of surgery on NK cell activity in an animal model. They studied marinating pulmonary NK cell activity and resistance to experimental tumor metastasis and the suppression by surgery and the reversal of suppression by the prophylactic use of a $\beta$-adrenergic antagonist and a prostaglandin synthesis inhibitor. This study confirmed the involvement of catecholamines and prostaglandins in the outcome of immune suppression. They showed that immune suppression was reversed by a prostaglandin synthesis inhibitor and a $\beta$-blocker. These findings support that potential prophylactic measures should be implemented in the cancer patient undergoing surgery. Baum, Demicheli, et al. [18] have reported that surgery unfavorably perturbs the natural history of early breast cancer by accelerating the appearance of distant metastases. It was felt that surgery could induce angiogenesis and proliferation of distant dormant micro-metastases, especially in node positive young patients. Other investigators $[19,20]$ have stressed the complex host-cancer relationship at the time of surgery. An example is the timing of surgery within the menstrual cycle, which may be an important factor regulating surgery induced angiogenesis for node positive premenopausal patients.

Little et al. [21] showed as early as 1993, that surgery and the perioperative period suppressed (CMI) and that interventions to reduce this risk would improve the long range outcome. Studies of surgical stress have confirmed the importance of NK cells in protection from metastasis. $\mathrm{Wu}$ and Lanier [22] have shown in animals that NK cells are important for cancer control especially with spread and growth of metastases. While Andersen et al. [23] reported that psychological and surgical stress suppress NK cell activity in humans and animals. However, and more importantly, some clinical studies have shown that levels of NK cell activity at the time of surgery predicts long term survival $[24,25]$. Riesco [26] has shown that the pretreatment total lymphocyte count predicts overall survival in cancer patients, independent of other major prognostic factors. Fumagalli et al. [27] have reported that lymphocyte counts independently predict overall survival in advanced cancer patients, and should be a biomarker for interleukin-2 (IL-2) immunotherapy.

There are several factors that are involved in the stress response to surgery and have been shown to inhibit NK cell activity in vitro. These agents are catecholamines, prostaglandins, and corticosteroids, which have been reported by Di Lorenzo et al. [28], and Dokur et al. [29]. These two groups have made a great contribution in this area, but Ben-Eliyahu et al. [30,31] and Melamed [17] have done tremendous animal work in surgical immune suppression. By using the MADB-106 tumor model in rats they implicated sympathetic responses in supporting metastasis during stress and surgery. They confirmed that B1 and B2 adrenoreceptor antagonists that blunt sympathetic responses reduced NK cell suppression and inhibited the increased susceptibility of experimental MADB106 metastasis that follow surgical stress, swim stress, social confrontation, and hypothermia. In vitro studies of Ellis et al. [32] and Whalen and Bankhurst [33] showed that catecholamines and prostaglandins suppress NK cell activity by elevating intracellular cyclic adenosine monophosphate (cAMP) levels.

The group from Israel continues to make great contributions to surgical and psychological NK cell Suppression. Rosenne et al. [34] sought to develop a clinically applicable preoperative regimen to prevent immunosuppression and promotion of metastasis by surgery and stress. They used the synthetic ds-RNA, poly I-C in vivo in F344 rats. It is thought that poly I-C protects immunocytes from suppression by (cAMP) elevating agents. Poly I-C increased the numbers of NK cells, while not increasing cytotoxicity, but profoundly protected marginating pulmonary NK cells from suppression by surgery. They felt that by increasing the numbers of marginating pulmonary NK cells, and increasing their resistance to immunosuppression, it could reduce postoperative metastasis in cancer patients.

It is important to think about these types of approaches to support host immunity very early in the cancer patient, especially during the stressful perioperative period. Most immunotherapeutic strategies attempt at boosting antitumor immunity but this is not enough to offset the immunosuppression of major surgery. Therefore, support during the immediate post-operative period is critical. The evidence suggest that boosting antitumor immunity and protecting immunity from postoperative suppression act via different cellular mechanisms combining both could be complimentary treatments and thus tremendously benefit the cancer patient. We [35] have shown that antitumor immunity can be accomplished with a mixed vaccine of autogenous and allogeneic breast cancer cells and tumor associated antigens CA15-3, CEA, and CA-125. The results in immune and clinical responses in breast cancer patients were reported. However, we believe it is time to implement competent antimetastatic measures, as it is critical in determining long-term survival.

It is now time for surgeons that treat cancer to start implementing competent antimetastatic immunity measures before, during, and after surgery. Host immunity in the perioperative period has been evaluated by the group in Israel, and again they have contributed much to our understanding of immune modulation during that period. 
Greenfield, Avraham, Benish, Goldfarb, et al. [36] studied immune suppression in patients while awaiting surgery and following it. They looked at dissociations between cytokine levels, their induced production, and NK cell cytotoxicity. They looked at the effects of various surgeries on a wide array of immune indices and compared patients' pre-operative immune status to control subjects. Blood procedures assessed NK cell number and cytotoxicity, and plasma cytokine levels and induced production of IFN $\gamma$, IL-6, IL-10, and IL-12. They found that surgery reduced NK cell numbers $/ \mathrm{mL}$ blood, pro-CMI cytokine production of IL-12 and IFN $\gamma$ were reduced by surgery. After surgery IFN $\gamma$ and IL-6 increased. Prior to surgery patients had impaired IL-12 induced production and NK activity with reduced plasma IFN $\gamma$ levels. Their findings showed that patients exhibit impaired immune function even before operation, which contributes to post-operative immune suppression. They believe that perioperative suppression of NK activity is mediated by neuroendocrine responses rather than Th1 cytokines.

Schwartz, Avraham, Benish, et al. [37] have shown that prophylactic IL-12 treatment reduces postoperative metastasis in a rat tumor model. The mediation of protection was by increased numbers but not cytotoxicity of NK cells. They found that IL-12 did not potentiate activity per NK cell nor protect it from suppression by surgery, but IL-12 increased the numbers of circulating and marginating pulmonary NK cells. Their study indicated that the antimetastatic effects of IL-12 are due to increased numbers of strategically located NK cells. They advocate IL-12 as a prophylactic approach against the potential metastasis promoting effects of surgery. Goldfarb, Benish, Rosenne, et al. [38] have shown that CpG-C oligodeoxynucleotides (CpG-C ODN) limit the deleterious effects of $\beta$-adrenoreceptors stimulation on NK cytotoxicity and metastatic dissemination. They believe prophylactic CPG-C ODN therapy can improve immunocompetence and potentially reduce metastatic dissemination in clinical settings characterized by enhanced sympathetic stress responses. We have recently shown that the cytolytic function of NK cells is inhibited by increased tumor iron concentration both intracellular and in the stromal microenvironment $[39,40]$.

\section{Conclusions}

The above evidence presented supports, without doubt, the concept of what I now call psychoneuro-immunooncology. It is important to continue research in cancer immunotherapy and establish it as the fourth modality of cancer therapy. The Big Three being surgery, chemotherapy, and radiation. I believe for cancer immunotherapy to be effective and accepted as the fourth modality of cancer treatment, it will take a very innovative combined approach. I have discussed and presented details about a combination cancer immunotherapy approach soon to be published [41]. However, I now truly believe for a combination cancer immunotherapy protocol to have the most opportune chance to be efficacious, we must address host immune support immediately at the time of diagnosis. By treating immune suppression associated with the psychic stress of diagnosis and surgical procedures, other cancer immunotherapeutic measures in the adjuvant and metastatic setting will probably be much more effective. This communication is an appeal to physicians that treat cancer especially surgeons, to begin to look at the host of the disease and beyond the cancer. It is definitely important to monitor and support host immunity at diagnosis, perioperative period and during adjuvant chemotherapy and radiation protocols. By addressing host immunity in all of these areas confronting cancer patients, we can offer patients better quality of life and possibly a better chance for survival. It is time for the cancer surgeon to think beyond the recommended surgical procedure for the disease and start the practice of psychoneuro-immunooncology.

\section{REFERENCES}

[1] R. L. Elliott and J. F. Head, "Host Immunity Ignored in Clinical Oncology: A Medical Opinion," Cancer Biotherapy and Radiopharmaceuticals, Vol. 20, 2005, pp. 123-125. doi:10.1089/cbr.2005.20.123

[2] I. J. Elenkov, R. L. Wilder, G. P. Chrousos and E. S. Vizi, "The Sympathetic Nerve-An Integrative Interface between Two Supersystems: The Brain and the Immune System," American Pharmaceutical Review, Vol. 52, 2000, pp. 595-638.

[3] S. Ben-Eliyahu, "The Promotion of Tumor Metastasis by Surgery and Stress: Immunological Basis and Implications for Psychoneuroimmunology," Brain, Behavior, and Immunity, Vol. 17, No. 1, 2003, pp. 27-36. doi:10.1016/S0889-1591(02)00063-6

[4] M. Urosevic and D. Reinhard, "Human Leukocyte Antigen-G and Cancer Immunoediting," Cancer Research, Vol. 68, No. 3, 2008, pp. 627-630. doi:10.1158/0008-5472.CAN-07-2704

[5] J. L. McCoy, R. Rucker and J. A. Petros, "Cell-Mediated Immunity to Tumor-Associated Antigens is a Better Predictor of Survival in Early Stage Breast Cancer than Stage, Grade or Lymph Node Status," Breast Cancer Research and Treatment, Vol. 60, No. 3, 2000, pp. 227-234. doi:10.1023/A:1006405504158

[6] R. L. Elliott, J. F. Head and J. L. McCoy, "Comparison of Estrogen and Progesterone Receptor Status to Lymphocyte Immunity against Tumor Antigens in Breast Cancer Patients," Breast Cancer Research and Treatment, Vol. 30, 1994, pp. 299-304. doi:10.1007/BF00665971

[7] J. F. Head, F. Wang, R. L. Elliott and J. L. McCoy, "Assessment of Immunologic Competence and Host Reactiv- 
ity against Tumor Antigens in Breast Cancer Patients: Prognostic Value and Rationale of Immunotherapy Development," Annals of the New York Academy of Sciences, Vol. 609, 1993, pp. 340-342.

doi:10.1111/j.1749-6632.1993.tb44024.x

[8] P. Eschwège, F. Dumas, P. Blanchet, V. Le Maire, G. Benoit, A. Jardin, B. Lacour and S. Loric, "Haematogenous Dissemination of Prostatic Epithelial Cells during Radical Prostatectomy," The Lancet, Vol. 346, 1989, pp. 1528-1530.

[9] K. Yamaguchi, Y. Takagi, S. Aoki, M. Futamura and S. Saji, "Significant Detection of Circulating Cancer Cells in the Blood by Reverse Transcriptase-Polymerase Chain Reaction during Colorectal Resection," Annals of Surgery, Vol. 232, No. 1, 2000, pp. 58-65. doi:10.1097/00000658-200007000-00009

[10] J. Folkman, "What is the Evidence that Tumors Are Angiogenesis Dependent," Journal of National Cancer Institution, Vol. 82, No. 1, 1990, pp. 4-6. doi:10.1093/jnci/82.1.4

[11] Br. Zetter, "Angiogenesis and Tumor Metastasis," Annual Review of Medicine, Vol. 49, 1998, pp. 407-424. doi:10.1146/annurev.med.49.1.407

[12] R. Abramovitch, M. Marikovsky, G. Meir and M. Neeman, "Stimulation of Tumor Growth by Wound-Derived Growth Factors," British Journal of Cancer, Vol. 79, No. 9-10, 1999, pp. 1392-1398. doi:10.1038/sj.bjc.6690223

[13] S. O. Hofer, G. Molema, R. A. Hermens, H. J. Wanebo, J. S. Reichner and H. J. Hoekstra, "The Effect of Surgical Wounding on Tumor Development," European Journal of Surgical Oncology, Vol. 25, No. 3, 1999, pp. 231-243. doi:10.1053/ejso.1998.0634

[14] R. L. Elliott, X. Jiang, J. Phillips, B. Barnett and J. F. Head, "Human Leukocyte Antigen G Expression in Breast Cancer: Role in Immunosuppression," Cancer Biotherapy and Radiopharmaceuticals, Vol. 26, No. 2, 2000, pp. 153-157. doi:10.1089/cbr.2010.0924

[15] C. Sietses, R. H. Beelen, S. Meijer and M. A. Cuesta, "Immunological Consequences of Laparoscopic Surgery, Speculations on the Cause and Clinical Implications," Langenbecks Archives of Surgery, Vol. 384, No. 3, 1999, pp. 250-258. doi: 10.1007/s004230050200

[16] E. Faist, C. Schinkel and S. Zimmer, "Update on the Mechanisms of Immune Suppression of Injury and Immune Modulations," World Journal of Surgery, Vol. 20, No. 4, 1996, pp. 454-459. doi:10.1007/s002689900071

[17] R. Melamed, E. Rosenne, K. Sakhar, Y. Schwartz, N. Abudarham and S. Ben-Eliyahu, "Marginating Pulmonary-NK Activity and Resistance to Experimental Tumor Metastasis: Suppression by Surgery and the Prophylactic Use of a Beta-Adrenergic Antagonist and a Prostaglandin Synthesis Inhibitor," Brain, Behavior, and Immunity, Vol. 19, 2005, pp. 114-126. doi:10.1016/j.bbi.2004.07.004

[18] M. Baum, R. Demicheli, W. J. M. Hrushesky and M. Retsky, "Does Surgery Unfavourably Perturb the "Natural History" of Early Breast Cancer by Accelerating the Appearance of Distant Metastases," European Journal of
Cancer, Vol. 41, 2005, pp. 508-515. doi:10.1016/j.ejca.2004.09.031

[19] W. J. M. Hrushesky, A. Z. Bluming, S. A. Guber and R. Sothern, "Menstrual Influence on Surgical Care of Breast Cancer," Lancet, Vol. 2, 1989, pp. 949-952. doi:10.1016/S0140-6736(89)90956-2

[20] K. Bove, D. W. Lincoln, P. A. Wood and W. J. M. Hrushesky, "Fertility Cycle Influence on Surgical Breast Cancer Cure," Breast Cancer Research and Treatment, Vol. 75, No. 1, 2002, pp. 65-72. doi:10.1023/A:1016543222323

[21] D. Little, M. Regan, R. M. Keane and D. Bouchier-Hayes, "Perioperative Immune Modulation," Surgery, Vol. 114, No. 1, 1993, pp. 87-91.

[22] J. Wu and L. L. Lanier, "Natural Killer Cells and Cancer," Advances in Cancer Research, Vol. 90, 2003, pp. 127-156. doi:10.1093/jnci/90.1.30

[23] B. L. Andersen, W. B. Farrar, D. Golden-Kreutz, L. A. Kutz, R. MacCallum, M. E. Courtney and R. Glaser, "Stress and Immune Responses after Surgical Treatment for Regional Breast Cancer," Journal of the National Cancer Institute, Vol. 90, No. 1, 1998, pp. 30-36. doi:10.1093/jnci/90.1.30

[24] A. Taketomi, M. Shimada, K. Shiabe, K. Kajiyama, T. Gion and K. Sugimachi, "Natural Killer Cell Activity in Patients with Hepatocellular Carcinoma: A New Prognostic Indicator after Hepatectomy," Cancer, Vol. 83, No. 1, 1998, pp. 58-63.

doi:10.1002/(SICI)1097-0142(19980701)83:1<58::AID-C $\underline{\mathrm{NCR} 8>3.0 . \mathrm{CO} ; 2-\mathrm{A}}$

[25] H. Takeuchi, Y. Maeharo, E. Tokunaga, T. Koga, Y. Kakeji and K. Sugimachi, "Prognostic Significance of Natural Killer Cell Activity in Patients with Gastric Carcinoma: A Multivariate Analysis," The American Journal of Gastroenterology, Vol. 96, No. 2, 2001, pp. 574-578. doi:10.1111/j.1572-0241.2001.03535.x

[26] A. Riesco, "Five-Year Cancer Cure: Relation to Total Amount of Peripheral Lymphocytes and Neutrophils," Cancer, Vol. 25, 1970, pp. 135-140. doi:10.1002/1097-0142(197001)25:1<135::AID-CNCR28 20250120>3.0.CO;2-9

[27] L. A. Fumagalli, J. Vinke, W. Hoff, E. Ypma, F. Brivio and A. Nespoli, "Lymphocyte Counts Independently Predict Overall Survival in Advanced Cancer Patients: A Biomarker for IL-2 Immunotherapy," Journal of Immunotherapy, Vol. 26, No. 5, 2003, pp. 394-402. doi:10.1097/00002371-200309000-00002

[28] G. Di Lorenzo, P. M. Esposito, A. Drago, P. Di Blasi, G. Candore, C. Balisteri, F. Listi and C. Caruso, "Effects of in vitro Treatment with Fluticasone Propionate on Natural Killer and Lymphokine-Induced Killer Activity in Asthmatic and Healthy Individuals," Allergy, Vol. 56, No. 4, 2001, pp. 323-327. doi:10.1034/j.1398-9995.2001.00879.x

[29] M. Dokur, N. Boyadjieva and D. K. Sarkar, "Catecholaminergic Control of NK Cell Cytolytic Activity Regulatory Factors in the Spleen," Journal of Neuroimmunology, 
Vol. 151, No. 1-2, 2004, pp. 148-157. doi:10.1016/i.jneuroim.2004.03.003

[30] S. Ben-Eliyahu, G. G. Page, R. Yirmiya and G. Shakhar, "Evidence that Stress and Surgical Interventions Promote Tumor Development by Suppressing Natural Killer Cell Activity," International Journal of Cancer, Vol. 8, No. 6, 1999, pp. 880-888. doi:10.1002/(SICI)1097-0215(19990315)80:6<880::AIDIJC14>3.0.CO;2-Y

[31] S. Ben-Eliyahu, G. Shakhar, G. G. Page, V. Stefanski and K. Shakhar, "Suppression of NK Cell Activity and of Resistance to Metastasis by Stress: A Role For Adrenal Catecholamines and Beta-Adrenoceptors," Neuroimmunomodulation, Vol. 8, No. 3, 2000, pp. 154-164. doi: $10.1159 / 000054276$

[32] N. K. Ellis, G. P. Duffie, M. R. Young and H. T. Wepsi HT, "The Effects of 16,16-Dimethyl PGE 2 and Phosphodiesterase Inhibitors on Con A Blastogenic Responses and NK Cytotoxic Activity of Mouse Spleen Cells," Journal of Leukocyte Biology, Vol. 47, No. 4, 1990, pp. 371-377.

[33] M. M. Whalen and A. D. Bankhurst, "Effects of Beta-Adrenergic Receptor Activation, Cholera Toxin and Forskolin on Human Natural Killer Cell Function," Biochemical Journal, Vol. 272, No. 2, 1990, pp. 327-331.

[34] E. Rosenne, G. Shakhar, R. Melamed, Y. Schwartz, A. Erdreich-Epstein and S. Ben-Eliyahu, "Inducing a Mode of NK-Resistance to Suppression by Stress and Surgery: A Potential Approach Based on Low Dose of Poly I-C to Reduce Postoperative Cancer Metastasis," Brain, Behavior, and Immunity, Vol. 21, No. 4, 2007, pp. 395-408. doi:10.1016/j.bbi.2006.12.002

[35] X. P. Jiang, D. C. Yang, R. L. Elliott and J. F. Head, "Vaccination with a Mixed Vaccine of Autogenous and Allogeneic Breast Cancer Cells and Tumor Associated
Antigens CA15-3, CEA and CA125-Results in Immune and Clinical Response in Breast Cancer Patients," Cancer Biotherapy and Radiopharmaceuticals, Vol. 15, No. 5, 2000, pp. 495-505. doi:10.1089/cbr.2000.15.495

[36] K. Greenfeld, R. Avraham, M. Benish, Y. Goldgarb, E. Rosenne, Y. Shapira, T. Rudich and S. Ben-Eliyahu, "Immune Suppression While Awaiting Surgery and Following It: Dissociations between Plasma Cytokine Levels, Their Induced Production, and NK Cell Cytotoxicity," Brain, Behavior, and Immunity, Vol. 21, 2007, pp. 503-513. doi:10.1016/i.bbi.2006.12.006

[37] Y. Schwartz, R. Avraham, M. Benish, E. Rosenne and S. Ben-Eliyahu, "Prophylactic IL-12 Treatment Reduces Postoperative Metastasis: Mediation by Increased Numbers but not Cytotoxicity of NK Cells," Breast Cancer Research and Treatment, Vol. 107, 2008, pp. 211-223. doi:10.1007/s10549-007-9540-9

[38] Y. Goldfab, M. Benish, E. Rosenne, R. Melamed, B. Levi, A. Glasner and S. Ben-Eliyahu, "CpG-C Oligodeoxynucleotides Limit the Deleterious Effects of Beta-Adrenoceptor Stimulation on NK Cytotoxicity and Metastatic Dissemination," Journal of Immunotherapy, Vol. 32, No. 2, 2009, pp. 280-291. doi:10.1097/CJI.0b013e31819a2982

[39] R. L. Elliott, X. P. Jiang and J. F. Head, "Reversal of Immune Suppression in Cancer by Manipulation of Tumor Iron Metabolism," Journal of Immunotherapy, Vol. 33, 2010, p. 869.

[40] X. P. Jiang, R. L. Elliott, B. G. Barnett and J. F. Head, "The Regulation of the Cytolytic Function Natural Killer Cells on the MCF-7 Human Breast Cancer Cell Line by Iron," AACR Special Conference on Tumor Immunology: Basic and Clinical Advances, Vol. 1, 2010, p. 101.

[41] R. L. Elliott, "Combination Cancer Immunotherapy "Expanding Paul Ehrlich's Magic Bullet Concept,” In Press. 